\title{
Searches for annihilating dark matter in the Milky Way halo with IceCube
}

\section{The IceCube Collaboration ${ }^{\dagger}$}

${ }^{\dagger}$ http://icecube.wisc.edu/collaboration/authors/icrc17_icecube

E-mail: samuel.d.flis@gmail.com

We present three searches for a neutrino signal from dark matter self-annihilations in the Milky Way. The signal from these events is identified by signatures which start developing within the fiducial volume of the IceCube Neutrino Observatory. The three presented analyses are sensitive to different energy scales and together cover an energy range from $10 \mathrm{GeV}$ to $300 \mathrm{TeV}$ in dark matter particle mass, while focusing on either track-like or cascade-like events. Two analyses incorporate energy in the analysis, while one analysis introduces a novel method to reconstruct events below $1 \mathrm{TeV}$. Experimental exclusion limits are presented for all analyses.

Corresponding authors: Samuel Flis*1, Morten Medici ${ }^{2}$

${ }^{1}$ Oskar Klein Centre and Dept. of Physics, Stockholm University, SE-10691 Stockholm, Sweden

${ }^{2}$ Niels Bohr Institute, University of Copenhagen, DK-2100 Copenhagen, Denmark

35th International Cosmic Ray Conference - ICRC2017

10-20 July, 2017

Bexco, Busan, Korea

* Speaker. 


\section{Introduction}

So far dark matter has only revealed its presence by its gravitational influence. Measurements of galactic rotation curves [1,2] and movements of galaxies in clusters such as the Coma cluster [3] indicate a much larger mass than could be accounted for by only observing the luminous matter. Currently our best description of the Universe is given by the $\Lambda \mathrm{CDM}$ [4] model in which cold nonbaryonic dark matter is a crucial ingredient. The nature of dark matter is still unknown, however it has to be fairly long lived given the current observational and experimental constraints, and it may interact weakly.

Many theories that extend the Standard Model predict particles with properties which fit the above description of dark matter. One of the most experimentally accessible dark matter candidates are the so-called Weakly Interacting Massive Particles (WIMPs) [5]. If these particles self-annihilate they might produce stable Standard Model particles, such as electrons, photons or neutrinos that are detectable at Earth. It is compelling to search for a neutrino signal from dark matter annihilation since neutrinos, which only interact weakly, propagate through the Universe largely unaffected. The direction and energy information that can be extracted from neutrinos will be unchanged from their creation even considering neutrino vacuum oscillations.

The rate of self-annihilation increases with the dark matter density making regions with presumably higher dark matter densities such as massive celestial objects, galaxies and dwarf galaxies interesting from an observational point of view. Galaxies are believed to be embedded in halos of dark matter that extend far beyond the optical disks of the galaxies. The differential neutrino flux from annihilating dark matter in the Milky Way assuming a dark matter particle mass, $m$, a neutrino energy spectrum from annihilating dark matter $\mathrm{d} N / \mathrm{d} E$ and a thermally averaged cross-section $\langle\sigma v\rangle$ is given by

$$
\frac{\mathrm{d} \Phi}{\mathrm{d} E}=\frac{\left\langle\sigma_{A} v\right\rangle}{4 \pi \cdot 2 m^{2}} \frac{\mathrm{d} N}{\mathrm{~d} E} \int \rho^{2}(r(l, \Psi)) \mathrm{d} l,
$$

where $\rho(r)$ is the dark matter density profile and the integral is evaluated along the line of sight. A variety of models try to describe the distribution of dark matter in galactic halos where spherical symmetry is often assumed. N-body simulations have commonly favored cuspy density profiles like the NFW profile which tend to diverge towards the center [6], while observations of low surface brightness galaxies suggest a flat (also called cored) distribution in the central region which in this work has been represented by the Burkert halo profile [7]. Figure 1 illustrates the two dark matter density profiles and how the line of sight integral, $J(\Psi)$, depends on the angle to the Galactic Center. Since it is still not clear whether the Milky Way halo is cuspy or cored we present results for both of the two halo profiles, using model parameter values from Ref. [8].

Three analyses are presented here, probing WIMP masses from $10 \mathrm{GeV}$ to $300 \mathrm{TeV}$ using neutrino data from the IceCube Neutrino Observatory[9]. IceCube instruments one cubic-kilometer of glacier ice at the geographical South Pole using 5160 detectors distributed on 86 strings. Neutrinos are detected by the Cherenkov radiation produced by secondary charged leptons created in neutrino interactions in the ice. Different event topologies (corresponding to different neutrino interactions) are used in the three analyses. Track-like events induced by muon neutrinos have good directional reconstruction in general, and are used in the first two analyses presented. The third 
analysis uses shower (cascade) events induced by charge-current interactions of electron and tau neutrinos as well as neutral current interactions from all neutrino flavors. Cascade events provide a better energy reconstruction at the price of poor pointing accuracy.
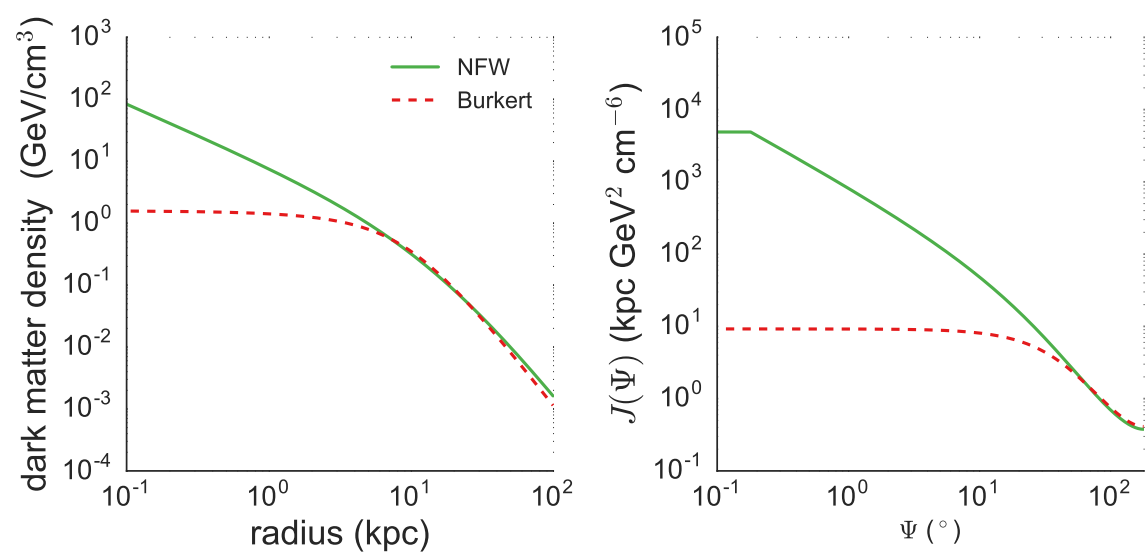

Figure 1: Left: Dark matter density profiles as a function of the distance to the center of the galaxy. Right: The line of sight integral for different profiles as a function of the angle to the center of the galaxy.

\section{Analysis method}

The analyses presented here use the same likelihood approach to search for a neutrino excess aligned with the Galactic Center that corresponds with dark matter annihilation in the Milky Way halo. The likelihood is defined as a mixture of signal, $S$, and background, $B$, and can generally be written down as

$$
\mathscr{L}(\xi)=\prod_{i}\left[\xi S\left(\theta_{i}\right)+(1-\xi) B\left(\theta_{i}\right)\right]
$$

where $\xi$ is the signal fraction and $\theta_{i}$ are the event observables. Signal is modeled by weighting simulated neutrino events with Eq. 1.1. The neutrino energy spectrum is determined from PYTHIA8 [10] simulation by forcing a generic resonance of twice the dark matter mass to decay into one of the considered dark matter annihilation channels. The background is estimated from experimental data, so will not be affected by systematic uncertainties from the simulation chain. However, due to the extended nature of the Milky Way halo, if a signal is present, the background estimation will be contaminated with signal. If the signal contamination is not accounted for it leads to biased estimators and a reduction of the analysis sensitivity. We counter the signal contamination by applying a signal subtraction method. The scrambled data is modeled as $\tilde{D}=\xi \tilde{S}+(1-\xi) B$ where the tilde denotes a scrambled quantity. Solving this for $B$ and putting it back into Eq. 2.1 yields the final form of the likelihood:

$$
\mathscr{L}(\xi)=\prod_{i}\left[\xi S\left(\theta_{i}\right)+\tilde{D}\left(\theta_{i}\right)-\xi \tilde{S}\left(\theta_{i}\right)\right] .
$$




\section{Low-Energy Galactic Center tracks}

A muon produced in a muon neutrino chargedcurrent interaction can travel many hundred meters through the ice. This allows for a good reconstruction of the neutrino direction, since it is well correlated to that of the muon. The dark matter analyses that are focused on muon track events are exploiting the pointing precision of the track to look for an anisotropy corresponding to the targeted signal from dark matter annihilation.

Below neutrino energies of $100 \mathrm{GeV}$, the muon tracks are more challenging to reconstruct, and are no longer clearly distinguishable from cascade events. However, with the improvement of IceCube event reconstruction, it has been possible to improve the sensitivity of IceCube to neutrino energies below $100 \mathrm{GeV}$. These improvements therefore make it possible for the low energy analyses to be sensitive to WIMP masses down to $10 \mathrm{GeV}$.

The event sample used in the low energy analysis is specifically designed for WIMP masses between $10 \mathrm{GeV}$ and $1 \mathrm{TeV}$, with details explained in Ref. [11]. The IceCube/DeepCore subdetector is used as the fiducial volume for the analysis, using the strings indicated in Figure 2, with detectors located between a depth of 2140-2420 m.

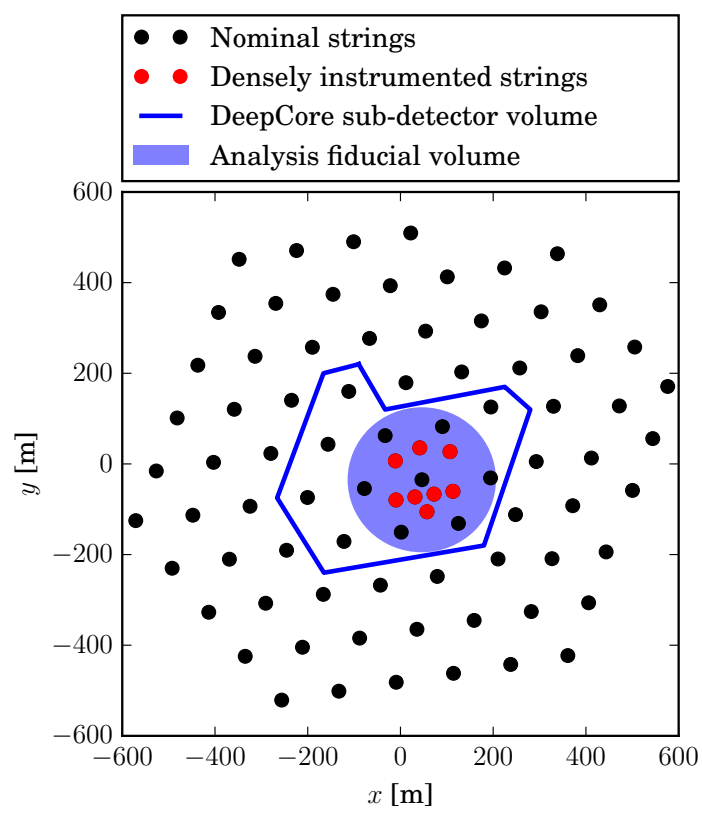

Figure 2: Fiducial volume of the sub-detector IceCube/DeepCore and the volume used for the analysis of the low energy galactic center search.

The remainder of the IceCube strings are used to tag and reject the massive background of down-going atmospheric muons. Muon neutrinos with a reconstructed arrival direction close to the center of the Milky Way in declination are primarily selected, however, interacting electron neutrinos and tau neutrinos (as well as neutral-current interactions) are also included.

In the analysis of the experimental data, all events are included if they have a reconstructed arrival direction in the full band of right ascension across the sky at a declination interval 2 radi- 
ans wide (centered on the galactic center). Since the atmospheric background is uniform in right ascension, most of the sensitivity of the analysis comes from studying this dimension.

The data were collected between 2012 and 2015 using the full 86-string configuration of IceCube with the sample containing 22632 events in total. In Figure 3 the declination bin covering the galactic center is presented, and the different expectations from the two dark matter halo models considered. Additionally this shows that no strong signal above the constant background was observed in data.

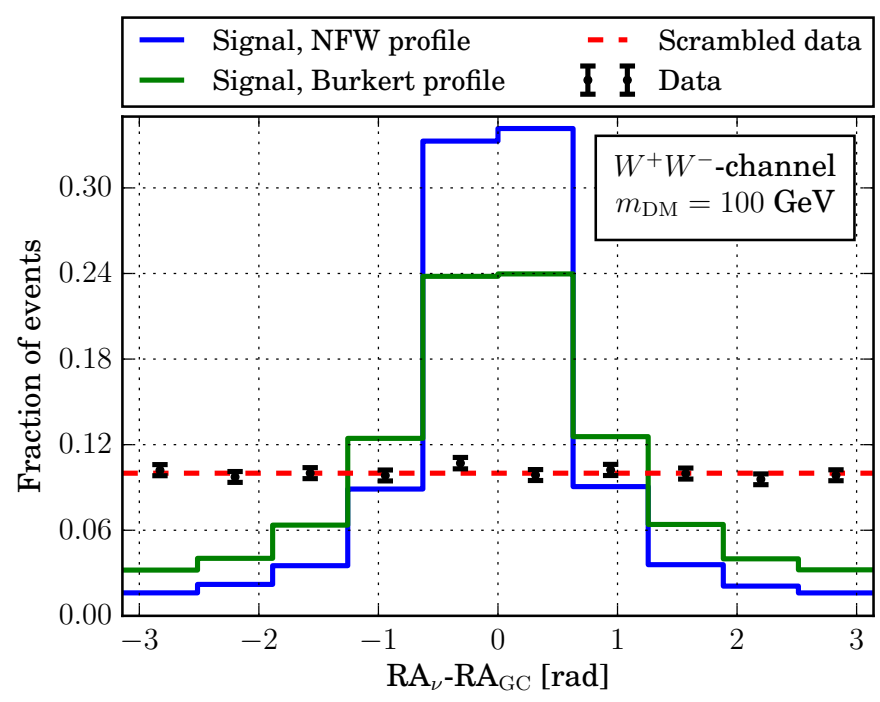

Figure 3: Distribution of events in right ascension for the low energy galactic center search, showing no excess above the flat background expected from atmospheric muons and neutrinos.

\section{High-Energy Galactic Halo tracks}

At higher energies the signal efficiency of muon neutrino induced through-going events grows faster than the efficiency to detect starting events. This analysis therefore used event samples with through-going events that traditionally were used for Point Source (PS) searches in IceCube [12, 13, 14]. Four years of IceCube data were used, spanning from the 40-string configuration of IceCube to the 86-string configuration. The PS datasets cover the full sky, however the atmospheric muon background is poorly suppressed in the southern hemisphere (the down-going region) compared to the northern hemisphere where the atmospheric muons are absorbed by Earth.

A dedicated point source neutrino sample for the southern hemisphere named Medium Energy Starting Events (MESE) [15] was also added. This sample consists of three years of data and the veto cut ensures a low muon background for the MESE sample. Even with the addition of the MESE sample an analysis on the combined sample is mostly sensitive in the northern hemisphere, and thus primarily probes the outer halo. An advantage of mainly probing the outer halo is the smaller dependence on the halo profiles which tend to converge at larger distances to the Galactic center. 
The analysis of the PS and MESE data samples was performed using an unbinned likelihood including both directional and energy observables. As the data samples were collected and processed under varying conditions, the background and signal was modelled specifically for each sample. In the end the likelihoods for each sample were combined into a joint likelihood.

\section{High-Energy Galactic Halo cascades}

This search uses starting events that are cascade-like, i.e. resembling electromagnetic or hadronic showers, presumably induced by neutrino interactions in the ice. Cascade events have the advantages of better energy reconstruction (due to the containment of the complete energy loss) and all-flavor sensitivity. The directional reconstruction is of course much worse compared to tracks, however, in the context of resolving the Milky Way halo which extends over the whole sky and in particular the Galactic Center region which covers several degrees, the effect of the reduced resolution of cascade events is mitigated. In contrast to the first IceCube dark matter search that used cascades [16], this search is focusing on relatively high WIMP masses.
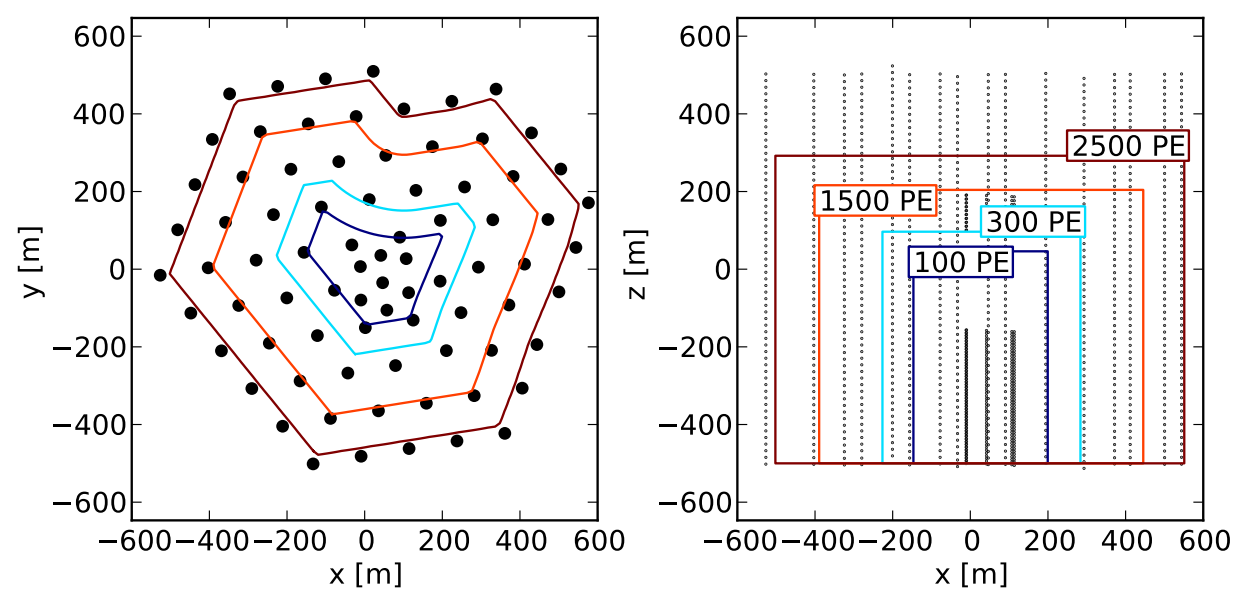

Figure 4: An illustration of how the fiducial volume grows with the detected deposited charge in a top down perspective (left) and a side view (right)

The event sample used in this analysis was originally developed for an unfolding analysis of different components of the neutrino spectrum observed at Earth [17]. The data were collected between 2010 and 2012 using one year of the 79-string configuration and the first year of the full 86-string configuration of IceCube. The events in the sample are required to start inside a fiducial volume which scales with the detected deposited charge of the event. Figure 4 illustrates how the fiducial volume scales for different amounts of deposited charge. The remaining volume serves as a veto region to reject incoming muons as well as distinguish between cascade events and outgoing track events. This analysis only considered events that were classified as cascades. In total the two samples contained 278 cascade events where 133 events were collected with the 79 -string detector and 145 events were collected with the 86-string detector. 
The data from the two years had separate geometries, calibrations, and separate simulation datasets, hence the two samples were treated separately. A binned likelihood analysis using energy and directional observables was performed. Similar to the previously described halo track analysis the likelihoods for the two samples were combined into a joint likelihood.

\section{Results and conclusions}

None of the analyses observed any excesses above the expectation from the background of atmospheric muons and atmospheric neutrinos, and instead limits on $\left\langle\sigma_{A} v\right\rangle$ were set. All analyses computed limits at $90 \%$ confidence according to Feldman and Cousins prescription [18]. Figure 5 shows the resulting limits assuming dark matter annihilation into tauons and an NFW profile (left panel) or a Burkert profile (right panel). Besides limits from the analyses presented here, limits from ANTARES and gamma ray telescopes are shown. Both the gamma ray experiments and the ANTARES analysis set limits that are lower than the IceCube limits for most of the mass range when assuming the NFW profile. However, when considering the Burkert profile, IceCube sets the most stringent limits along most of the mass range. IceCube analyses probe the full sky or at least a large portion of the sky and are therefore less sensitive to variations between profiles in the central region of the Galactic Center. Probing a large part of the sky is also advantageous in the case of extended profiles such as the cored Burkert halo where $90 \%$ of the signal is contained within $10^{\circ}-15^{\circ}$ in the sky. This is best illustrated with the high-energy galactic halo track search (IC 4yr PS) that essentially only probes the outer halo and produces very similar limits for both profiles.
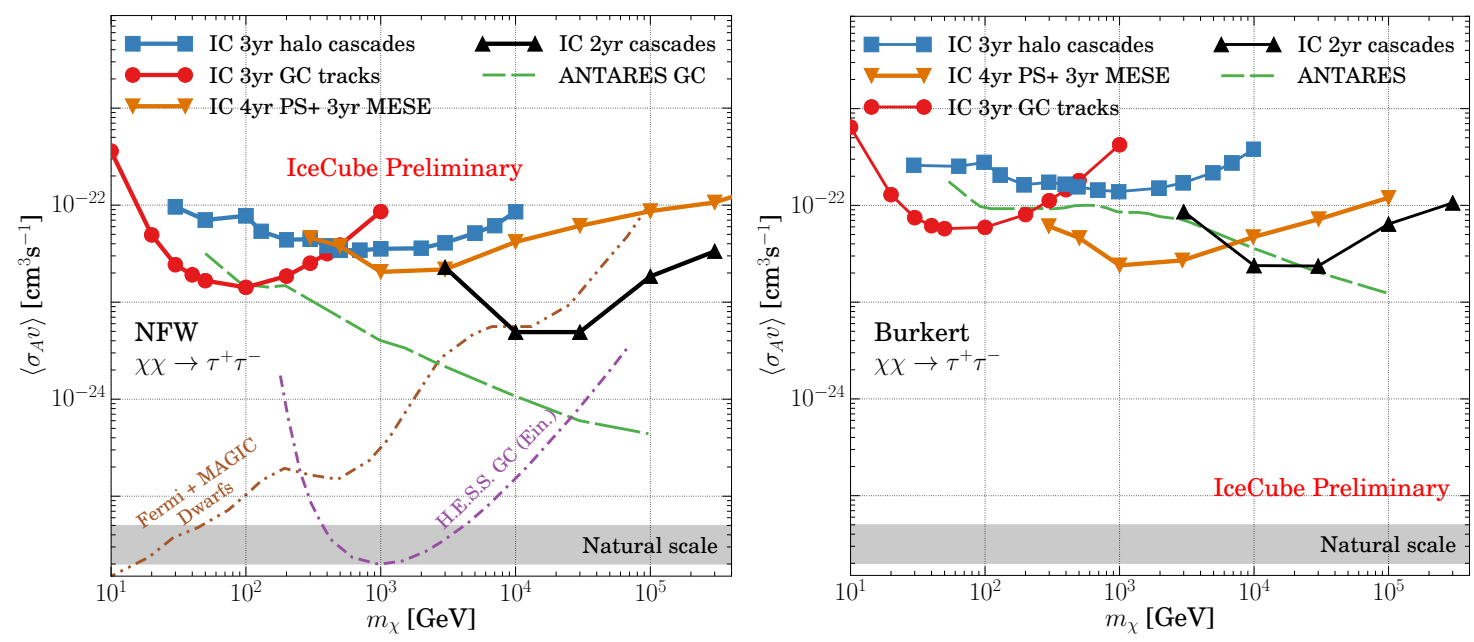

Figure 5: Comparison of upper limits on $\left\langle\sigma_{A} v\right\rangle$ versus WIMP mass, for the annihilation channel $\chi \chi \rightarrow$ $\tau^{+} \tau^{-}$, assuming the NFW profile (left) and the Burkert profile (right). The analyses presnted here are compared to ANTARES [19] and the IceCube 3yr halo search [16]. Also shown are upper limits from gamma-ray searches from the dwarf galaxy Segue 1 (Seg1) by FermiLAT+MAGIC [20] and the Galactic Center by H.E.S.S. [21]. The natural scale refer to the value of $\langle\sigma v\rangle$ that is needed for WIMPs to be a thermal relic [22] 


\section{References}

[1] H. W. Babcock, Lick Observatory Bulletin 19 (1939) 41

[2] V. C. Rubin and Jr. W. K. Ford, Astrophys. J. 159 (1970) 379

[3] F. Zwicky, Helvetica Physica Acta 6 (1933) 110

[4] Planck Collaboration, P. A. R. Ade et al., Astron. Astrophys. 594, A13 (2016)

[5] J. E. Gunn, B. W. Lee, I. Lerche, D. N. Schramm and G. Steigman, Astrophys. J. 223 (1978) 1015

[6] J. F. Navarro, C. S. Frenk and S. D. M. White, Astrophys. J. 462 (1996) 563

[7] A. Burkert, Astrophys. J. 447 (1995) L25

[8] F. Nesti and P. Salucci, JCAP 1307 (2013) 016

[9] IceCube Collaboration, M. G. Aartsen et al., JINST 12 (2017), P03012

[10] T. Sjostrand, S. Mrenna and P. Z. Skands, Comput. Phys. Commun. 178 (2008) 852

[11] IceCube Collaboration, M. G. Aartsen et al., arXiv:1705.08103 [hep-ex].

[12] IceCube Collaboration, R. Abbasi et al., Astrophys. J. 732 (2011) 18

[13] IceCube Collaboration, M. G. Aartsen et al. Astrophys. J. 779 (2013) 132

[14] IceCube Collaboration, M. G. Aartsen et al. Astrophys. J. 796 (2014) 109

[15] IceCube Collaboration, M. G. Aartsen et al., Astrophys. J. 824 (2016) L28

[16] IceCube Collaboration, M. G. Aartsen et al., Eur. Phys. J. C 76 (2016) 531

[17] IceCube Collaboration, M. G. Aartsen et al., Phys. Rev. D 91 (2015) 022001

[18] G. J. Feldman and R. D. Cousins, Phys. Rev. D 57 (1998) 3873

[19] A. Albert et al., Phys. Lett. B 769 (2017) 249

[20] MAGIC and Fermi-LAT Collaborations, M. L. Ahnen et al., JCAP 1602 (2016) 039

[21] H.E.S.S. Collaboration, H. Abdallah et at., Phys. Rev. Lett. 117 (2016) 111301

[22] G. Steigman, B. Dasgupta, and J. F. Beacom, Phys. Rev. D 86 (2012) 023506 